\title{
Single-electron transport through stabilised silicon nanocrystals
}

\begin{abstract}
Tuhin Shuvra Basu, (D)* Simon Diesch (D) and Elke Scheer (D)
We have fabricated organically capped stable luminescent silicon nanocrystals with narrow size distribution by a novel, high yield and easy to implement technique. We demonstrate transport measurements of individual silicon nanocrystals by scanning tunnelling microscopy at a low temperature in a doublebarrier tunnel junction arrangement in which we observed pronounced single electron tunnelling effects. The tunnelling spectroscopy of these nanocrystals with different diameters reveals quantum confinement induced bandgap modifications. Furthermore, from the features in the tunnelling spectra, we differentiate several energy contributions arising from electronic interactions inside the nanocrystal. By applying a magnetic field, we have detected a variation in the differential conductance profile that we attribute to arising from higher order tunnelling processes. We have also systematically simulated our experimental data with the Orthodox theory, and the results show good agreement with the experiment. The study establishes a correlation between the nanocrystal size and quantum confinement induced band-structure modifications which will pave the way to devise tailored nanocrystals.
\end{abstract}

\section{A. Introduction}

Investigation of electronic transport through nanostructured materials at the single-particle level is an important area of research for exploring the fundamental physics of a small system and for the construction of advanced electronic devices, e.g., single electron transistors (SETs). ${ }^{1,2}$ Recently, single-particle transport measurements of semiconductor nanocrystals (NCs) were performed by scanning tunnelling spectroscopy (STS) in a controlled but non-alterable microelectronic system where the sample has been fabricated by sophisticated in situ lithography. ${ }^{3}$ The complex fabrication process restricts the application potential of the NCs because of the difficulty in integrating them into existing device concepts. Therefore, separate preparation of the NCs and subsequent transfer to the tunnelling microscope would be advantageous. In this way, their properties in relation to their size can be studied systematically. Silicon (Si) NCs belong to the most important classes of semiconductor NCs with multifaceted application possibilities. They exhibit strong room temperature photoluminescence (PL) ${ }^{4}$ due to the excitonic recombination of the quantum confinement induced widened bandgap. ${ }^{5}$ They also have potential applications in optoelectronic devices, ${ }^{6}$ in the renewable energy sector, ${ }^{6,7}$ and even in bio-

Department of Physics, University of Konstanz, 78457 Konstanz, Germany. E-mail: tuhin.basu@uni-konstanz.de logical imaging/tagging. ${ }^{8}$ Si NC integration in Si-based microelectronics is of increasing importance in non-linear optics for photonic communication ${ }^{9}$ though much-advanced technology is still required to extract the maximum efficiency. ${ }^{10}$ Thus, a proper understanding of their properties at the most fundamental single-particle level can open up new unexplored possibilities. In this regard, the STS study of individual Si NCs is the method of choice to determine their local density of states (LDOS). When brought into weak contact with electrodes, a Si NC exhibits pronounced single electron tunnelling (SET) phenomena. ${ }^{11}$ However, comprehensive experimental studies of the single electron transport behaviour of Si NCs are still sparse. There are few reports on vapour-deposited and grown Si NCs in which the transport characteristics through degenerate states of the conduction band of individual NCs were studied by STS, ${ }^{12-15}$ though this topic still warrants a more systematic study to build robust knowledge.

In this work, we extensively characterise the electronic transport and bandgap modifications of individual hexyl-functionalised Si NCs at a very low temperature $(300 \mathrm{mK})$ in a double-barrier tunnel junction (DBTJ). ${ }^{16}$ The hexyl-functionalisation of the Si NCs not only prevents the NCs from oxidising but avoids unnecessary complexity arising from other surface functionalisation methods. Our findings support quantum confinement effects of Si NCs in a single-particle measurement. We have successfully correlated the features appearing in the STS with the NC size and surface functionalisation. In the DBTJ arrangement, the transport of the electrons is largely governed by the coupling capacitances and the resistances of 
the tunnel junctions. ${ }^{17-20}$ We have calculated these parameters by electrostatic considerations and have investigated their influence on the STS features. From STS we have successfully explained and assigned the quasi-particle energy gap $\left(E_{\mathrm{g}}^{\mathrm{qp}}\right)$, the single-particle bandgap $\left(E_{\mathrm{g}}^{\mathrm{sp}}\right)$ and the self-interacting coupling energy between the electrons of individual functionalised $\mathrm{Si}$ NCs by employing a semi-classical approach. Furthermore, we have observed a band variation in Si NCs during the tunnelling process by applying a moderate magnetic field. The experimental findings have also been validated by the Orthodox theory.

\section{B. Optical and structural characterisation}

From the same batch of hexyl-capped Si NCs which is used to prepare samples for the STS measurements, we performed optical and structural characterisation measurements.

The steady-state and time-resolved PL of the background corrected hexyl-capped Si NCs has been measured by using a FluoTime 300. The steady-state PL spectrum is shown in Fig. 1a and the time-resolved data are provided in the inset of Fig. 1a. The Si NC ensemble exhibits strong room temperature PL due to the excitonic recombination of the quantum confinement induced widened pseudo-direct bandgap. ${ }^{4,5}$ Fig. 1a shows a PL peak at $492 \mathrm{~nm}$, suggesting an energy gap of 2.5 eV. The Gaussian profile with a smaller FWHM-width suggests a narrow size distribution of the Si NC ensemble. Importantly we did not see any PL peak due to oxide related states, ${ }^{21-23}$ typically appearing at a longer wavelength and hence we believe that the ensemble of Si NCs is oxide-free.
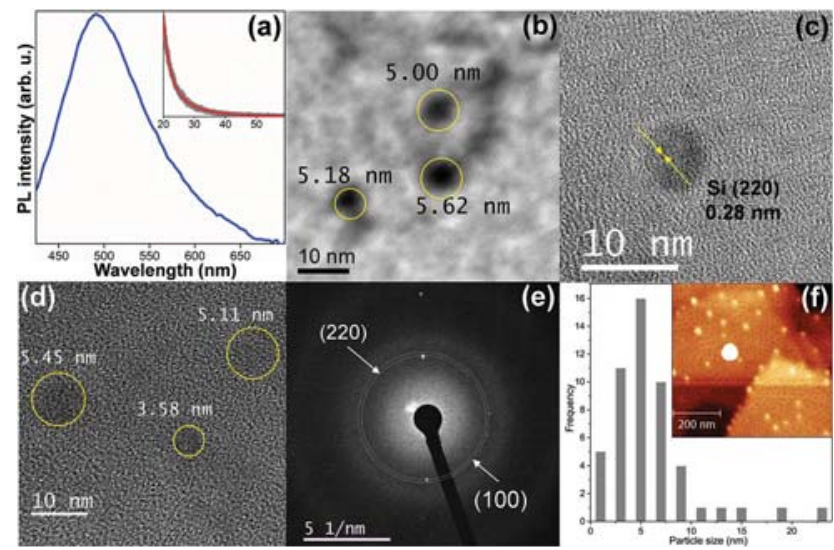

Fig. 1 (a) PL emission of the Si NC ensemble shows a peak at $492 \mathrm{~nm}$. The inset shows the time-resolved PL emission with an average decay time 3.26 ns. (b) Three Si NCs with diameter $\sim 5 \mathrm{~nm}$. (c) Free-standing Si $\mathrm{NC}$ with a fringe width of $0.28 \mathrm{~nm}$ corresponding to the Si (220) plane. (d) Three different Si NCs of diameter 3-5 nm. (e) SAEDP of $d$. (f) Histogram of the size dispersion of Si NCs on the prepared substrate (inset). One exceptionally big blob seen in the inset is probably due to the unwanted agglomeration of Si NCs or contamination with dust.
The excitation source for time-resolved PL measurements was a picosecond pulsed laser diode of $405 \mathrm{~nm}$. Furthermore, we have calculated the decay dynamics of the same sample and the decay time has been analyzed by the time-correlatedsingle-photon-counting (TCSPC) technique. The multi-component fitting has been performed with a PicoQuant FluoFit by using the following equation and is shown as a red line in the inset picture.

$$
I(t)=\sum_{i=1}^{n} A_{i} e^{-\frac{t}{\tau_{i}}} \quad(n=3)
$$

The above equation suggests that the excited electrons have chosen three decay channels during radiative recombination to the ground state. One decay $\left(\tau_{1}\right)$ has a lifetime of $7.14 \mathrm{~ns}$ with $37 \%$ contribution. The other two decays have much shorter lifetimes of 1.84 ns and 0.05 ns with $33 \%$ and $30 \%$ contribution, respectively, to the overall intensity. The average lifetime of the Si NC ensemble is 3.26 ns. From the above results, it is evident that some of the electrons radiatively recombine to the ground state directly with a longer lifetime (7.14 ns), whereas the shorter lifetimes (1.84 and $0.05 \mathrm{ns)}$ ) are due to dominant non-radiative faster decay channels originating from charge trapping at the surface-mediated defect states occurring due to the organic ligand attachment to the Si NC surface. ${ }^{24}$ The phenomenon is pronounced in the single-particle measurement of a $4.2 \mathrm{~nm} \mathrm{Si} \mathrm{NC} \mathrm{(Fig.} 2$ and 4) where we have observed two interface states inside the single-particle gap of the Si NC.
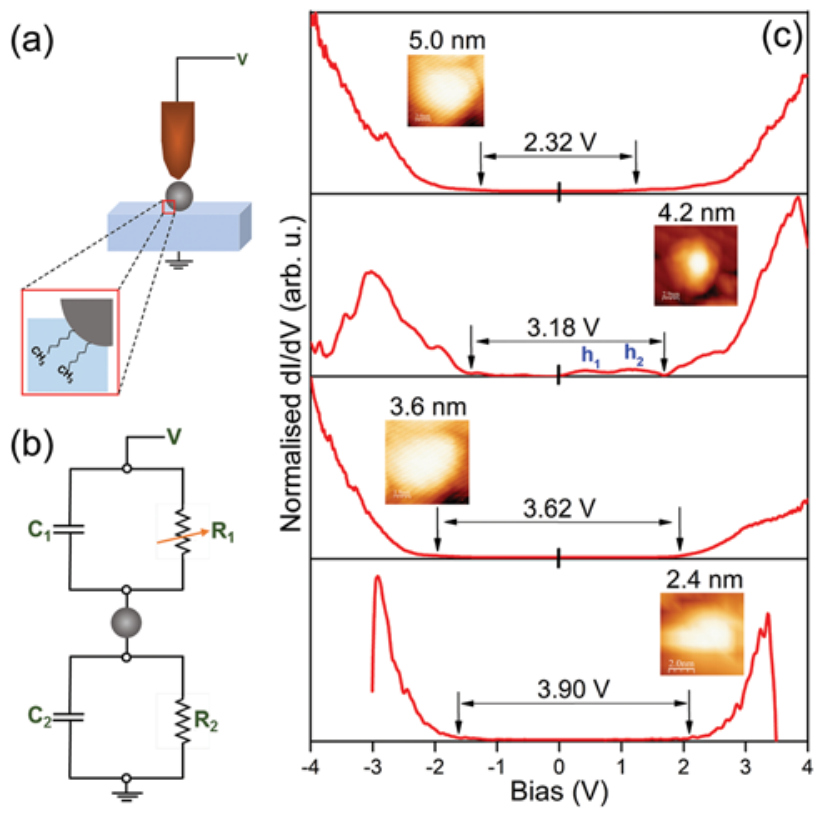

Fig. 2 (a) Visualisation of hexyl-capped Si NCs in DBTJ arrangement. The magnified image shows the anchoring of the hexyl-group to the Au substrate. (b) Equivalent electrical circuit of DBTJ. (c) Differential conductance of individual Si NCs with different sizes. The arrows indicate the transition from zero to non-zero differential conductance and the numbers below the images indicate the size of the zero-conductance gaps. 
The structural characterisation has been performed by high-resolution transmission electron microscopy (HR-TEM) at $200 \mathrm{kV}$ acceleration voltage. To this end, $3 \mu \mathrm{L}$ of the as-synthesised Si NCs, dispersed in hexane, were deposited on a $2 \mathrm{~nm}$ thin $\mathrm{C}$-coated $\mathrm{Cu}$ grid and were imaged in the brightfield mode. The results of the structural characterisation are provided in Fig. 1b-e. HR-TEM images (1b-d) show Si NCs with the dimension of 3-5 $\mathrm{nm}$ (Fig. $1 \mathrm{~b}$ and d). The reason for having low contrast is the comparable atomic weight of the $\mathrm{C}$ grid of the HR-TEM and bulk Si. Nevertheless, we recorded images of some free-standing Si NCs. One of the representative images is shown in Fig. 1c. We measured the fringe width, and the value $(0.28 \mathrm{~nm})$ corresponds to the interplanar distance of the Si (220) planes. The nominal increase in the interplanar distance from the bulk Si (220) planes $^{25}$ is possibly due to the stress exerted by the hexyl-terminal of Si NCs. Similar phenomena have been observed in other NC systems. ${ }^{26,27}$ Fig. 1e shows the selected area electron diffraction pattern (SAEDP) of the bright field image of Fig. 1d. Two rings with two different diameters are characteristic of the $\mathrm{Si}$ (100) and (220) planes, respectively, ${ }^{25,28}$ and are coming from two Si NCs. For the crystal plane calculation from the SAEDP, we have considered the stress exerted by the hexyl-terminal of Si NCs. Thus, this study confirms the fabrication of crystalline Si NCs that will be further utilised in the single-particle measurement.

The inset of Fig. 1f shows the distribution of the Si NCs on the prepared substrate. The histogram of the atomic force microscopy (AFM) image shows the presence of a majority of particles in the range $\leq 5 \mathrm{~nm}$, though bigger particles are also observed. Diameters of all Si NCs have been calculated from the height-profile analysis with the software package WSXM. ${ }^{29}$

The optimum concentration of hexyl-capped Si NCs was $11.8 \mu \mathrm{g} \mathrm{l}^{-1}$ or $0.012 \mathrm{ppm}$ to obtain the uniform dispersion shown in Fig. 1f (see the ESI $\dagger$ for the calculation).

\section{Single electron tunnelling through individual Si NCs}

In this section, we will discuss the electronic transport through individual Si NCs using a scanning tunnelling microscope (STM) in a DBTJ configuration. Fig. 2a shows the scheme of a DBTJ when a functionalised Si NC is placed between the STM tip and the Au substrate, and Fig. $2 \mathrm{~b}$ is the equivalent electronic circuit consisting of a series of two resistances and capacitances. ${ }^{30}$ We report STS of four individual Si NCs with a diameter of 2.4, 3.6, 4.2 and $5.0 \mathrm{~nm}$ at $300 \mathrm{mK}$. The corresponding differential conductance of the NC is shown in Fig. 2c. We only consider particles having a size $<5 \mathrm{~nm}$ for pronounced confinement effects.

In each differential conductance curve $(\mathrm{d} I / \mathrm{d} V)$, we have observed a zero-conductance gap (ZCG) and thereafter an exponential rise in both positive and negative bias. We have estimated the ZCG by the intersection between the averaged zeroconductance region and the onset of the exponential rise. This ZCG is proportional to the single-particle gap of the Si NC and the polarisation (charging) energy related to an image charge developed on the surface of the NC due to the dielectric discontinuity of the NC core and its functionalised shell.

For further analysis it is important to know the type of tunnelling occurring in our system. We argue that in our case the tunnelling-out of the electrons from the NC to the substrate is faster than the tunnelling-in from the tip to the NC. This situation is known as the shell-tunnelling condition. ${ }^{31}$ In the opposite case (shell-filling condition), the electronic charge would reside inside the Si NC for a while resulting in coulombic repulsion of the incoming tunnelling electrons. In the conductance curve, we should then observe equi-spaced multiple peaks (with a peak width equal to the charging energy) according to the degeneracy of the states. We rule out the possibility of shell-filling conditions due to the absence of any pronounced multiple peaks in all $\mathrm{d} I / \mathrm{d} V$ s shown in Fig. 2c. We conclude that the conductance spectrum solely reflects the singleparticle LDOS and that the ZCG is proportional to the Si NC single-particle gap plus the polarisation (charging) energy. In the tunnelling spectra (Fig. 2c), the size of the observed ZCG $\left(\Delta V_{0}\right)$ is indicated by black arrows. We have detected that this gap is widening inversely with the NC size. However, there is a difference between the observed ZCG in the experimental conductance spectrum and the actual gap in the LDOS of the system. The gap in the LDOS is modified by a proportionality factor, $\eta$, which is equal to $\frac{C_{2}}{C_{1}+C_{2}}$ and is reflected in the experimental tunnel spectra. ${ }^{31}$ In our case, the values of $\eta$ vary from $0.506,0.509,0.510$ to 0.512 for $2.4,3.6,4.2$ and $5.0 \mathrm{~nm}$, respectively (see the ESI $\uparrow$ for the calculation of the capacitances $C_{1}$ and $C_{2}$ ). The values of $\eta$ suggest a slightly higher voltage drop across the tip-NC than across the NC-substrate junction and cause minor modifications in the actual gap compared to the experimentally observed ZCG. In order to extract the single-particle bandgap $\left(E_{\mathrm{g}}^{\mathrm{sp}}\right)$ of individual Si NCs (in the shell-tunnelling condition) from the observed ZCG $\left(\Delta V_{0}\right)$, we have employed the following equation: ${ }^{32}$

$$
\eta \Delta V_{0}=E_{\mathrm{g}}^{\mathrm{qp}}=E_{\mathrm{g}}^{\mathrm{sp}}+2 \Sigma
$$

$E_{\mathrm{g}}^{\mathrm{qp}}$ is termed the quasi-particle gap (in the LDOS) and $E_{\mathrm{g}}^{\mathrm{sp}}=$ $\varepsilon_{1}^{\mathrm{e}}-\varepsilon_{1}^{\mathrm{h}}$, where $\varepsilon_{1}^{\mathrm{e}}$ and $\varepsilon_{1}^{\mathrm{h}}$ are the lowest electron and hole energy levels related to the single-particle spectrum in the neutral state. $\Sigma(r)$ is the interaction energy of the electrons and expressed as the energy polarisation of the NC's own image charge at the surface of the functionalised Si NC. It depends on the dielectric inequality of the Si NC core and the organic ligand shell. $\Sigma(r)$ for a NC of radius $r$ and the dielectric constant $\varepsilon_{\mathrm{nc}}$ when surrounded by another material of dielectric constant $\varepsilon_{\text {out }}$ is: ${ }^{33}$

$$
\Sigma(r) \approx \frac{1}{2}\left(\frac{1}{\varepsilon_{\mathrm{out}}}-\frac{1}{\varepsilon_{\mathrm{nc}}}\right) \frac{e^{2}}{r}+\delta \Sigma(r)
$$

As

$$
\frac{\varepsilon_{\mathrm{nc}}}{\varepsilon_{\mathrm{out}}} \gg 0, \delta \Sigma(r) \approx 0.47 \frac{e^{2}}{\varepsilon_{\mathrm{nc}} r}\left(\frac{\varepsilon_{\mathrm{nc}}-\varepsilon_{\mathrm{out}}}{\varepsilon_{\mathrm{nc}}+\varepsilon_{\mathrm{out}}}\right)
$$


The dielectric constant of NCs, $\varepsilon_{\mathrm{nc}}$, is size dependent. We have employed the following relationship to extract $\varepsilon_{\mathrm{nc}} \cdot{ }^{34,35}$

$$
\varepsilon_{\mathrm{nc}}(r)=1+\frac{\varepsilon_{\mathrm{b}}-1}{1+\left(\frac{1.38}{2 r}\right)^{1.37}}
$$

$\varepsilon_{\mathrm{b}}$ is the dielectric constant of the bulk crystalline $\mathrm{Si}$ and it amounts to 11.4. The values are 8.1, 9.2, 9.5 and 9.9 for the

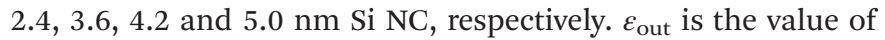
the dielectric constant of the capping agent of the Si NC (2.0).

Thus, the calculated polarisation (charging) energies are $0.226,0.156,0.135$ and $0.115 \mathrm{eV}$ for $2.4,3.6,4.2$ and $5.0 \mathrm{~nm} \mathrm{Si}$ NCs, respectively.

The observed quasiparticle gap with the different diameters of Si NCs can be expressed by the following scaling law:

$$
E_{\mathrm{g}}^{\mathrm{qp}}-E_{\mathrm{g}, \mathrm{bulk}}^{\mathrm{qp}} \approx \frac{K_{1}}{(2 r)^{1.28}}
$$

$K_{1}$ is a scaling constant. Eqn (5) is formulated by assuming that $E_{\mathrm{g}}^{\mathrm{qp}}$ of Si NCs has a size-dependent deviation from the constant bulk quasiparticle gap $\left(E_{\mathrm{g}, \mathrm{qu}}^{\mathrm{qp}}\right)$ and changes inversely with the NC's diameter. The value of the scaling constant (here 1.28) has been determined from the fitting parameters of the experimental values shown in Fig. 3 by grey squares. Employing eqn (2) and (3), we have calculated $E_{\mathrm{g}}^{\text {sp }}$ of all Si NCs shown in Fig. 3 (blue squares). We conclude that there is a clear, confinement-induced single-particle bandgap widening of individual Si NCs with decreasing the NC dimension. In order to check the consistency of our data, we have compared

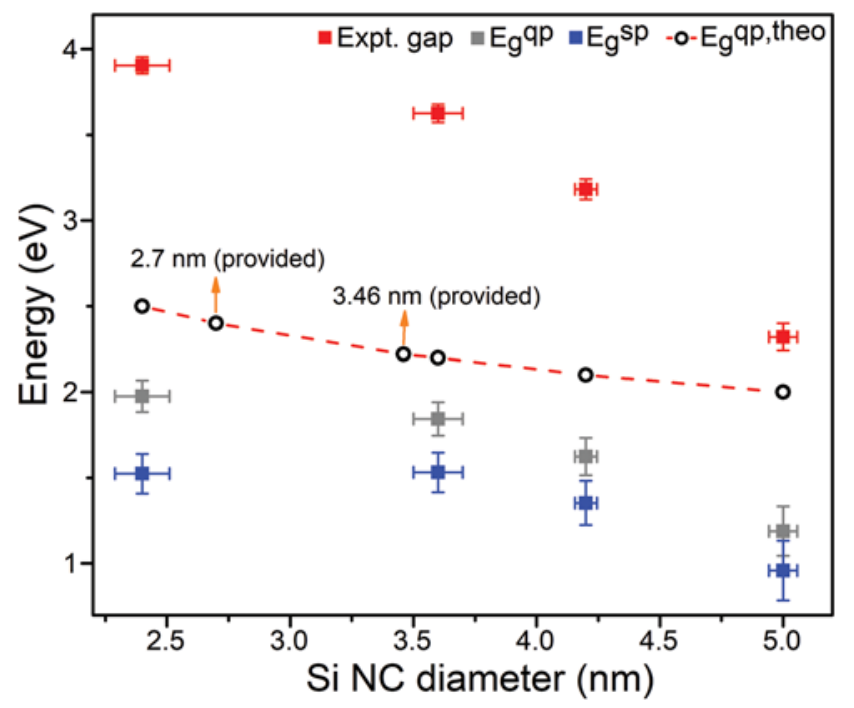

Fig. 3 Energy mapping of Si NCs obtained from the experiment and theory. The red squares are the values of the experimental zero-conductance gap obtained from Fig. 2c. After normalisation they are coined as $E_{\mathrm{g}}^{\mathrm{qp}}$ and are indicated by grey squares. For the validation of our data, we have compared them with the theoretically calculated quasi-particle gap (indicated by hollow circles). Furthermore, $E_{\mathrm{g}}^{\mathrm{sp}}$ values have been marked by blue squares. Vertical error bars represent the STS measurement uncertainty, whereas the horizontal error bars provide the Si NC size uncertainty. our $E_{\mathrm{g}}^{\mathrm{qp}}$ with a similar system ${ }^{36}$ where $E_{\mathrm{g}}^{\mathrm{qp}}$ of $\mathrm{H}$-passivated $\mathrm{Si}$ NCs has been calculated by a pseudopotential approach (indicated by hollow black circles in Fig. 3). The approach provides an accurate description of the wave-function attenuation outside the NC and it also accounts for inter-band coupling due to confinement. Though in that work the authors have

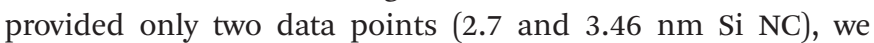
have extrapolated their data by applying a scaling law similar to eqn (5) with a different proportionality factor indicated by the red dashed line in Fig. 3. We observe good agreement with the experimental data with an offset of $\sim 0.5 \mathrm{eV}$ from the experimental values (grey squares). In our experiment, we have used hexyl-capped Si NCs, whereas the theoretical calculation has been performed with H-passivated Si NCs. Hexyl capping around the Si NC can induce a blue-shift in $E_{\mathrm{g}}^{\mathrm{sp}}\left(E_{\mathrm{g}}^{\mathrm{qp}}\right)$ compared to the H-terminated Si NC due to the tensile strain developed in the $\mathrm{Si} \mathrm{NC}$ as a similar phenomenon has been previously observed in $\mathrm{CH}_{3}$-terminated $\mathrm{Si} \mathrm{NC}^{37}$ The change in the organic ligand around the Si NC can be the cause of this deviation. In this context, it is important to discuss the effect of interface states (interface traps) ${ }^{38}$ between the organic ligand (capping agent of the Si NC) and the Au substrate. It has been observed that the chain length of the ligand plays an important role in determining the interface states. It has been detected that when the $\mathrm{C}$ chain length of the ligand to an $\mathrm{Ag}$ surface increases beyond $0.5 \mathrm{~nm}$, the effect of the energy shift due to the interface states is negligible. ${ }^{39}$ In our case, the chain length is $0.92 \mathrm{~nm}$ and being a similar material $(\mathrm{Au})$, we therefore assume that there is no energy shift due to interface traps.

The differential conductance characteristics of the $4.2 \mathrm{~nm}$ Si NC shown in Fig. 2c and 4a exhibit several significant features: (i) inside the gap region $\left(b_{1} \sim b_{2}\right)$, between the black arrows, we observe two conductance maxima (marked as $h_{1}$ and $h_{2}$ ) at positive bias, (ii) above the second increase of the conductance at the gap edge, zero-conductance is retrieved at higher bias, and (iii) strong conductance peaks arise outside the ZCG. We interpret these maxima as a result of higher order tunnelling processes. In this case, electrons are allowed to tunnel via an intermediate virtual state where first order tunnelling would be suppressed inside the ZCG. This inelastic cotunnelling in DBTJ is rare but not unusual. ${ }^{40,41}$ The tentative energy scheme is depicted in Fig. $4 \mathrm{~b}$. According to the scheme (Fig. 4b), first, two electrons hop from the STM tip to two surface-mediated (with energies $\Delta E_{1}$ and $\Delta E_{2}$ above the ground state) excited states (inside the bandgap) of the Si NC. At the same time, two other electrons residing in the ground state of the Si NC will tunnel out to occupy the same energy states available in the Au substrate. The extra energy has been provided by the applied bias. This process occurs at $e V_{\text {bias }}=$ $\Delta E_{1}$ or $\left(\Delta E_{1}+\Delta E_{2}\right)$ as shown in Fig. $4 \mathrm{~b}$. It results in two finite non-zero conductance regions within the ZCG (marked by $\mathrm{h}_{1}$ and $h_{2}$ in Fig. $2 c$ and $4 a$ ). Usually, non-zero conductance due to co-tunnelling appears symmetrically around zero, but we did not see that feature in the negative bias. We attribute this asymmetry to the asymmetry of the couplings of the NC to the 
(a)
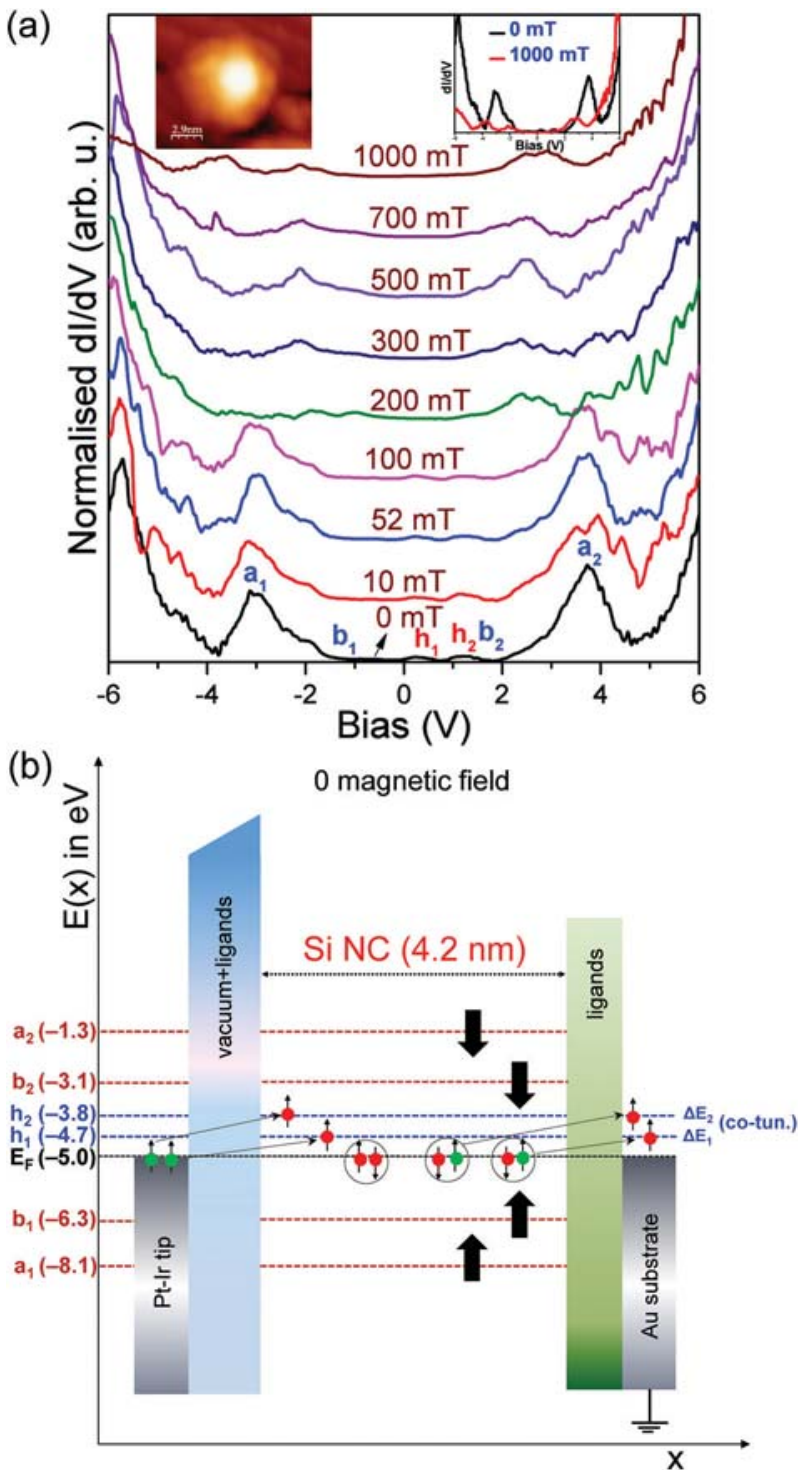

Fig. 4 (a) Variation of STS by applying a cross-plane magnetic field on a $4.2 \mathrm{~nm}$ Si NC. (b) The electronic transport scheme is depicted by an energy diagram at zero-magnetic-field. All values are provided in accordance with the STS. The black arrows show the movement of individual states with the increasing magnetic field. In addition, the blue dashed lines, corresponding to co-tunnelling processes, will disappear in the higher magnetic field.

tip and to the substrate, such that the inverse processes are suppressed. In addition, we observed a decrease in conductance at an interval of $3.6 \mathrm{eV}$ (the real value after normalisation is $1.8 \mathrm{eV}$ ) outside the STS gap $\left(\mathrm{a}_{1}\right.$ and $\mathrm{a}_{2}$ in Fig. 4a). These peaks may arise from the inclusion of an excited electronic state rather than a higher charge state.

In order to further explore the above scenario, we have investigated the development of the tunnel spectra with the magnetic field from 0 to $1 \mathrm{~T}$ in the out-of-plane direction. For field strengths up to $100 \mathrm{mT}$, the spectra remain unchanged. Above $200 \mathrm{mT}$, the co-tunnelling peaks are mainly suppressed and the $a_{1}$ and $a_{2}$ peaks are flattened and shifted together. During the field sweep, the overall tunnelling resistance remains constant, meaning that also the overall tunnelling resistances to the tip and the substrate can be assumed to be unaltered. These findings are tentatively explained as follows: the field dependence of the $a_{1}$ and $a_{2}$ peak rules out the interpretation of these peaks as Coulomb staircase peaks, but suggests a field-dependence of the electronic states involved in the transport. Zeeman effects can also safely be neglected for the field strengths applied here ${ }^{42}$ as for Si NCs there is only a $1.5 \mathrm{meV}$ shift in the LDOS gap due to the increase in the magnetic field from 0 to $11 \mathrm{~T}^{43}$ The suppression of co-tunnelling at a higher field $(200 \mathrm{mT})$ can be explained by spin-dependent transport during the co-tunnelling process. Recently, a pronounced magnetoresistance in small fields has been observed for molecular layers incorporating $\mathrm{Au}$-thiol bonds. ${ }^{44}$ This magnetoresistance was explained as arising from different electron transfer rates for the spin singlet and spin triplet. The proposed mechanism resulting in different rates is not specific to the Au-thiol bond, though. We thus argue that a similar mechanism might be at play here. The ground state of the NC is a spin singlet state, whereas the excited state is a spin triplet state. ${ }^{45}$ We argue that the presence of the tip breaks the spherical symmetry and thereby lifts the spin degeneracy of the tunnelling rates. For the co-tunnelling processes to occur, the coupling rates of the spin states with both electrodes must be finite. When applying a magnetic field, the coupling strength of these states with one of the electrodes (presumably the $\mathrm{Au}$ substrate) might be altered. As a result, the probability of the co-tunnelling transition through these excited states vanishes with the application of a magnetic field (here beyond $200 \mathrm{mT}$ ). Therefore, in Fig. $4 \mathrm{~b}$, the blue dashed line corresponding to co-tunnelling processes will disappear. Besides, we have observed a clear field-dependent quenching of $E_{\mathrm{g}}^{\mathrm{qp}}$ (hence $E_{\mathrm{g}}^{\mathrm{sp}}$ ) when the magnetic field changes from 100 to $200 \mathrm{mT}$. The phenomenon is clearly evident from the positions of the $b_{1}$ and $b_{2}$ peaks as they occur at lower bias values when the field changes from $100 \mathrm{mT}$ to $200 \mathrm{mT}$. The absolute value of $\left(\mathrm{b}_{1} \sim \mathrm{b}_{2}\right)$ provides the quasi-particle gap, $E_{\mathrm{g}}^{\mathrm{qp}}$. Consequently, differential conductance peaks due to the conduction of electrons through excited electronic states will also shift toward zero, as shown in Fig. 4a. The process has been depicted by the black arrows in Fig. $4 \mathrm{~b}$. One can argue that the reduction of $\left(b_{1} \sim b_{2}\right)$ and the consequent shift of $a_{1}$ and $a_{2}$ towards the low bias have occurred simply due to the availability of the lower energy levels for the electron transport beyond $100 \mathrm{mT}$. We have safely discarded this possibility, because then the transition of the differential conductance curve from $100 \mathrm{mT}$ to $200 \mathrm{mT}$ would not be smooth (as seen in Fig. 4a). One would thus expect an entirely dissimilar differential conductance profile with the appearance of new characteristic peaks. Concluding this part, the observed magnetic field dependence of the conductance spectrum suggests that $E_{\mathrm{g}}^{\mathrm{sp}}$ and the tunnelling processes can be tuned by the application of an external magnetic field, for this specific system. Hence, we argue that one can use this phenomenon to construct a magnetically 
switchable single electron transport device by altering the size of the NC and changing the core and shell material.

\section{Simulation with the Orthodox theory}

According to the Orthodox theory, the tunnelling rate for the $i$ th junction is $\Gamma_{i}^{ \pm}(n)$, where \pm suggests either the 'tip-to-NC' or 'NC-to-tip' tunnelling process across the junctions $(n \rightarrow n \pm 1) .^{30}$

$$
\Gamma_{i}^{ \pm}(n)=\left(\frac{1}{R_{i} e^{2}}\right)\left[\frac{-\Delta E_{i}^{ \pm}}{1-e^{\left(\Delta E_{i}^{ \pm} / k_{\mathrm{B}} T\right)}}\right]
$$

Here, $\Delta E_{i}^{ \pm}$is the change in energy when the electron tunnels through the barrier. The tunnelling rate $\Gamma_{i}^{ \pm}(n)$ is inversely proportional to the total tunnelling resistance. Now considering the electrostatic energy in each junction $(e>0)$, eqn (6) turns out to be:

$$
\begin{aligned}
& \left.\Delta E_{1}^{ \pm}=\left(\frac{e}{C_{1}+C_{2}}\right)\left[\frac{e}{2} \pm\left(n e-Q_{0}\right) \mp C_{2} V\right)\right] \\
& \left.\Delta E_{2}^{ \pm}=\left(\frac{e}{C_{1}+C_{2}}\right)\left[\frac{e}{2} \pm\left(n e-Q_{0}\right) \pm C_{1} V\right)\right]
\end{aligned}
$$

$Q_{0}$ is the fractional residual charge inside the NC and $Q_{0}(\bmod e)$ can be determined by the following equation..$^{30,46}$

$$
Q_{0}=\frac{1}{e}\left[C_{2}\left(\Delta \Phi_{2}\right)-C_{1}\left(\Delta \Phi_{1}\right)\right]
$$

$\Delta \Phi_{1}$ and $\Delta \Phi_{2}$ are the contact potentials across junctions 1 and 2.

Thus the fractional residual charges of the $\mathrm{Si} \mathrm{NC}$ with dimensions of $2.4 \mathrm{~nm}, 3.6 \mathrm{~nm}, 4.2 \mathrm{~nm}$ and $5.0 \mathrm{~nm}$ are $0.15 e$, $0.24 e, 0.29 e$ and $0.34 e$, respectively.

The collective distribution of the electrons on the NC, considering the overall probability of electron tunnelling between two adjacent states in the steady state is zero, can be described as:

$$
\sigma(n)\left[\Gamma_{1}{ }^{+}(n)+\Gamma_{2}{ }^{+}(n)\right]=\sigma(n+1)\left[\Gamma_{1}^{-}(n+1)+{\Gamma_{2}}^{-}(n+1)\right]
$$

where $\sigma(n)$ is the ensemble distribution of the number of electrons on the central electrode. Since $\Gamma_{i}^{ \pm}$can be evaluated from eqn (6) and (7), $\sigma(n)$ can be re-written in terms of the tunnelling current $I(V)$ using the normalisation condition $\sum_{n=-\infty}^{\infty} \sigma(n)=1$ :

$$
\begin{aligned}
I(V) & =e \sum_{n=-\infty}^{\infty} \sigma(n)\left[\Gamma_{2}^{-}(n)-\Gamma_{2}^{+}(n)\right] \\
& =e \sum_{n=-\infty}^{\infty} \sigma(n)\left[\Gamma_{1}^{+}(n)-\Gamma_{1}^{-}(n)\right]
\end{aligned}
$$

From the $I(V)$ curves, we calculate the differential conductance by numerical derivation.
We carried out the numerical analysis with the Simon 2.0 single electron device and the circuit simulator. This simulator is a Monte Carlo simulator for single electron circuits and devices, which can address the rate equation of any customisable design. In this simulation, we have to insert several parameters, e.g., tunnelling resistances, tunnelling capacitances, background charge, and type of material. The values of the capacitances are estimated electrostatically and are provided in the ESI. $\dagger$ The background charges are already calculated by eqn (8). The type of material we have chosen is a semiconductor with the experimental bandgap value. But the choice of tunnelling resistances is not straightforward. The methodology to find them will be discussed in the following paragraph.

As discussed earlier, the tunnelling resistances provide the tunnelling rates, $\Gamma_{i} \propto \frac{1}{R_{i}}(i=1,2)$. As in all of our cases $\Gamma_{1}<\Gamma_{2}$ (shell-tunnelling condition), the electron-electron interaction will be suppressed and a resonant tunnelling peak occurs due to the transport of single electrons (one by one) through the Si NC. Unlike the other parameters, the two tunnelling resistances, $R_{1}$ and $R_{2}$, at junctions 1 and 2 cannot be measured independently. We cannot use the classical tunnelling equation; e.g., the resistance-vs.-distance relationships to predict the resistances due to the organic ligand shell surrounding the NC. This ligand alters the work function on which the classical tunnelling equation is based. Thus, our approach is to determine the resistance ratio of the two junctions on which the profile of the curve is greatly dependent. The variation of the ratio will introduce modifications and/or additional features in the STS profile outside the LDOS gap. By matching the STS profile and its small non-linearities between the simulated and experimental curves, we chose the matching resistance ratio for each measurement. Taking into account the total resistance, one obtains an estimate for the individual resistance. ${ }^{30}$ Some of the sharp peaks in the experimental curves might have been caused by a certain change in the STM tip and therefore cannot be compared with the theoretical data. The changes may arise from sporadic noise from the electronic set-up causing a large output signal though that occurs extremely rarely. Actually, these kinds of noise are nullified during averaging over several spectra.

The comparison between simulated and experimental data is shown in Fig. 5a-d. The experimental curves are broadened in nature (except Fig. 5a, which fitted well) compared to simulated data due to the non-linearities of the differential conductance of the Au-coated mica surface. This usually occurs when the applied bias voltage (here maximum applied bias is $6 \mathrm{~V}$ ) is comparable to the work function of the substrate (work function of the Au substrate under vacuum is $5.1 \mathrm{eV}) .{ }^{47}$ Besides, in the experiment, the effective geometry and the tunnel parameters may vary due to the variation of the STM set point or the temperature during the measurement and can therefore introduce broadening. The simulation also assumes a rigid Coulomb-island, whereas experimentally hexyl-functionalised Si NCs are flexible in nature.

However, we know the total resistance, $R_{\mathrm{T}}$, from the STM set-up parameter, and it was $10 \mathrm{G} \Omega$ for the $2.4 \mathrm{~nm} \mathrm{Si} \mathrm{NC}$ 

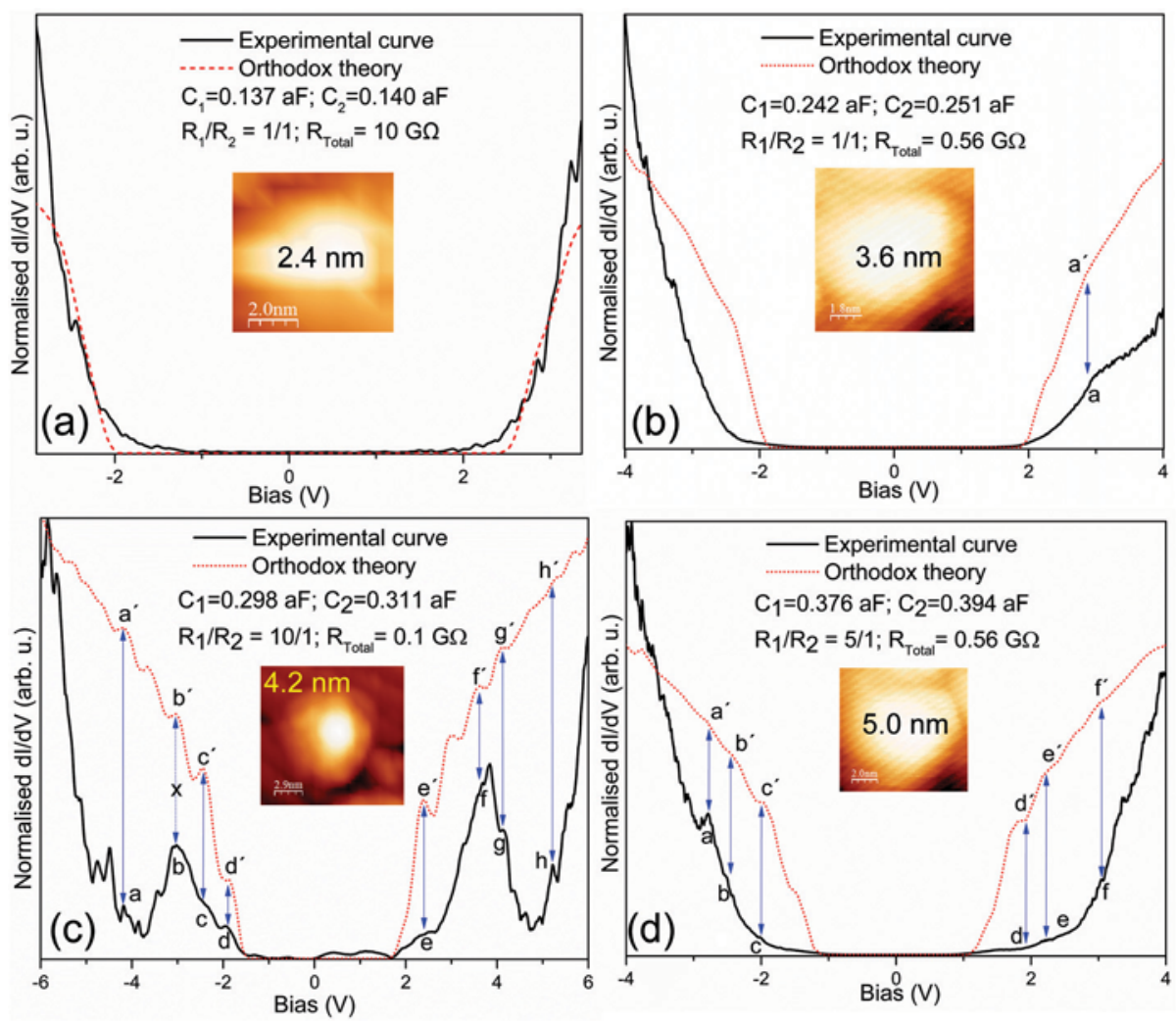

Fig. 5 Comparison of simulations with the Orthodox theory and experimental curves of (a) $2.4 \mathrm{~nm}$, (b) $3.6 \mathrm{~nm}$, (c) $4.2 \mathrm{~nm}$ and (d) $5.0 \mathrm{~nm}$ Si NCs.

(Fig. 5a). We have performed the simulation using the ratio $R_{1} / R_{2}=1$ and it perfectly matches with the experimental STS. For the $3.6 \mathrm{~nm}$ Si NC (Fig. $5 \mathrm{~b}), R_{\mathrm{T}}(0.56 \mathrm{G} \Omega)$ is also distributed in a $1 / 1$ ratio. The most astonishing resemblance has been observed for the $4.2 \mathrm{~nm}$ Si NC where $R_{\mathrm{T}}(0.1 \mathrm{G} \Omega)$ is distributed in a 10/1 ratio (Fig. 5c). As mentioned above, this curve deviates from the others by additional features, partially inside the ZCG which have been marked by a ( $\left.a^{\prime}\right)$ to $h\left(h^{\prime}\right)$ in the experimental (simulated) curve. Some of the peaks are more pronounced in the experimental curve than in the theoretical one and may thus be due to physics that is not covered by the simplified model used in the simulation. We have also marked all the steps of Fig. 5 d, where $R_{\mathrm{T}}(0.56 \mathrm{G} \Omega)$ is distributed in a $5 / 1$ ratio. Thus this study reveals that the ratio of the tunnelling resistances plays a key role in the shape of the STS. Thus, simulated STS can give valuable insights into the electronic transport through individual Si NCs.

\section{E. Si NC fabrication}

Hexyl-functionalised Si NCs have been fabricated following a method described elsewhere, ${ }^{48,49}$ but modified according to our requirements. In a typical assay, $350 \mu \mathrm{L}$ trichloro(hexyl) silane $\left[\mathrm{SiCl}_{3}-\mathrm{CH}_{3}\left(\mathrm{CH}_{2}\right)_{5}\right]$ and $200 \mu \mathrm{L}$ silicon tetrachloride $\left(\mathrm{SiCl}_{4}\right)$ were added into $200 \mathrm{~mL}$ dry toluene slowly using a micro-syringe and then sonicated for $35 \mathrm{~min}$ for uniform mixing. Trichloro(hexyl)silane serves both as a surfactant and as a reactant. It forms a self-assembled layer around $\mathrm{SiCl}_{4}$ in dry toluene resulting in a reverse micelle structure with a $\mathrm{SiCl}_{4}$ core and a trichloro(hexyl)silane shell (Scheme 1).

We have prepared a solution separately with an excess amount of lithium aluminium hydride $\left(\mathrm{LiAlH}_{4}\right)$ powder $(1 \mathrm{M})$ in dry tetrahydrofuran (THF). The mixture was added dropwise (very slowly) by using a micro-syringe into the previously grown micelle structure followed by a homogenisation for $45 \mathrm{~min}$. $\mathrm{LiAlH}_{4}$ has reduced the reverse micelle by a vigorous reaction and the reaction resulted in the formation of stable Si NCs with hexyl-groups covering the surface. The reaction was then quenched by the addition of excess dry ethanol and the reaction mixture was evaporated under reduced pressure which produces a grey powder-like substance.

$20 \mathrm{~mL}$ hexane has been added to dissolve the grey powder containing Si NCs and was further washed with $60 \mathrm{~mL}$ deionised (DI) water. The immiscible organic layer was passed

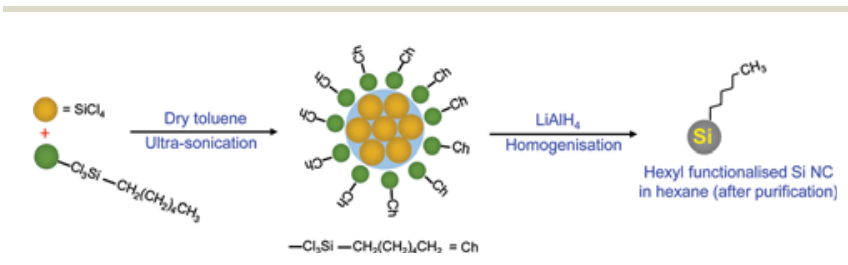

Scheme 1 One-pot synthesis of hexyl-functionalised Si NCs. 
through a polyvinylidene fluoride (PVDF) membrane with $0.22 \mu \mathrm{m}$ pore size to avoid agglomerated portions. Finally, a welldispersed luminescent hexyl-functionalised NC with narrow size distribution has been obtained in hexane. The entire procedure was performed under an inert gas $\left(\mathrm{N}_{2}\right)$ atmosphere inside a glovebox to prevent unwanted oxidation. During the whole process, the $\mathrm{O}_{2}$ level inside the glove-box was controlled to stay $<10.0 \mathrm{ppm}$. All the chemicals were procured commercially and, except for the solvents, were used as purchased. All dry solvents were obtained through a solvent purification system with an extremely low ppm $(<20 \mathrm{ppm})$ level of oxygen and moisture.

\section{F. Preparation of samples with Si NCs for STM}

The scanning region for tunnelling measurements is limited to an area of about $1 \mu \mathrm{m} \times 1 \mu \mathrm{m}$ by the design of our STM. ${ }^{50}$ In the present study, we have scanned individual areas as small as $50 \mathrm{~nm} \times 50 \mathrm{~nm}$ because of the limited scan speed and the small size of the NCs. NCs have to be well distributed and well attached to the substrate in order to find them within the smallest scan area. The substrate we used here was an atomically flat $\mathrm{Au}(111)$ thin film deposited on mica commercially procured from PHASIS Sàrl, Switzerland. A monolayer of Si NCs was prepared by immersing the $\mathrm{Au}(111)$ film vertically for $12 \mathrm{~h}$ into a Si NC-rich hexane solution inside the glovebox. Immobilised $\mathrm{Si}$ NCs stuck on the surface with an overall uniform distribution. The sample was further annealed at $70{ }^{\circ} \mathrm{C}$ for $12 \mathrm{~min}$ onto a hot-plate to evaporate excess hexane. Then we performed AFM in the non-contact mode with a $10 \mathrm{~nm}$ radius Si tip to capture the overall spatial distribution (see Fig. 1f). The prepared substrate was then transferred to the STM chamber which was brought to cryogenic vacuum.

\section{G. Set-up parameters during STM imaging and STS}

STM imaging and STS are performed with a Pt-Ir tip in a custom-made low temperature STM mounted in a ${ }^{3} \mathrm{He}$ cryostat at $300 \mathrm{mK}$. The set-up was built and refined by previous group members. ${ }^{50,51}$ The STM controller used was a commercially available SPM1000, by RHK Technology with special low-noise current amplifier (IVP-300). We performed slow $x$-direction scanning to achieve low-noise topography images with $256 \times$ 256 data points. The topography images were recorded in a constant current mode with the STM feedback loop set to a tunnelling current of $50-150 \mathrm{pA}$ at $1.5-5 \mathrm{~V}$ bias voltage. Current-voltage characteristics in the tunnelling regime were recorded by positioning the STM tip above an individual Si NC. During spectroscopy, the feedback loop was tuned to a higher time constant and a lower gain parameter to maximise the stability of the tip, and later, throughout the data acquisition, the feedback loop was switched off. The bias voltage was modulated with ac voltage, with a root-mean-square ampli- tude of $40 \mathrm{mV}$ and a frequency of $733 \mathrm{~Hz}$ for $\mathrm{d} I / \mathrm{d} V$ spectra via a lock-in technique. The $\mathrm{d} I / \mathrm{d} V$ was recorded by using a current-to-voltage converter with $1 \mathrm{~V} \mathrm{nA}^{-1}$ gain and the measuring time was $30 \mathrm{~s}$ per spectra. Set up current and voltage have been varied from 1.0-20.0 nA and 3.0-5.0 V, respectively, to achieve a good stability of the tip during data acquisition. The $\mathrm{d} I / \mathrm{d} V$ spectra, shown in this article, are obtained by averaging subsequent repetitive spectra. STS measurements have also been performed with an external magnetic field varying from $0-1 \mathrm{~T}$ in a cross-plane direction. ${ }^{50}$ We have observed clear SET behaviour as the total tunnelling resistance $\left(R_{\mathrm{T}}\right)$ in our experiment is much larger $(100 \mathrm{M} \Omega-$ $10 \mathrm{G} \Omega$ ) than the quantum resistance $\mathrm{h} / \mathrm{e}^{2}=25.8 \mathrm{k} \Omega$. The thermal energy smearing $\left(k_{\mathrm{B}} T\right)$ at $300 \mathrm{mK}$ (experimental temperature) amounts to $0.026 \mathrm{meV}$ and is much lower than the charging energy $\Sigma(r)$ of a typical Si NC $(0.226 \mathrm{eV})$.

\section{H. Conclusions}

In summary, we have successfully fabricated hexyl-functionalised stable luminescent Si NCs with a size $<5 \mathrm{~nm}$. We have constructed robust DBTJs with a single functionalised Si NC placed in an STM operating at a very low temperature of $300 \mathrm{mK}$. Individual tunnelling spectroscopy exhibits pronounced Coulomb blockade of the electronic transport through the Si NCs. The systematic STM study probes a quantum confinement induced bandgap increment when reducing the diameter of the NC. We have also shown that besides the intrinsic properties of the Si NC, the STS strongly depends on the suitable choice of the junction parameters. The careful analysis of the STS including calculating all tunnelling parameters, by combining simple electrostatic and quantum tunnelling equations, enables us to reveal different energy contributions to the conductance behaviour of single $\mathrm{Si}$ NCs. In addition, we have observed a variation in the differential conductance of a particular Si NC by applying a weak magnetic field ranging from 0 to $1 \mathrm{~T}$. The result suggests that the electronic structure of the ligands can be tailored by the magnetic field and that single NCs may be operated as magneticfield driven switches in future. We also validate our data with the well-established Orthodox theory. This knowledge can be later used to choose exact DBTJ parameters for stable STS irrespective of the chosen NC material.

\section{Conflicts of interest}

There are no conflicts to declare.

\section{Acknowledgements}

We thank A. Zumbusch, K. Hauser and J. Boneberg for giving access to their lab for characterisation measurements and for helpful discussion. We are indebted to M. Krumova for HRTEM imaging. We are grateful to the Deutsche 
Forschungsgemeinschaft for partial funding of the project in the framework of SFB767. T. S. B. acknowledges financial support from the Zukunftskolleg of the University of Konstanz and from the Alexander von Humboldt Foundation. We greatly thank Andreas Donges for assisting us to prepare the artwork.

\section{Notes and references}

1 K. Maeda, N. Okabayashi, S. Kano, S. Takeshita, D. Tanaka, M. Sakamoto, T. Teranishi and Y. Majima, ACS Nano, 2012, 6, 2798-2803.

2 M. Taupin, E. Mannila, P. Krogstrup, V. F. Maisi, H. Nguyen, S. M. Albrecht, J. Nygård, C. M. Marcus and J. P. Pekola, Phys. Rev. Appl., 2016, 6, 054017.

3 S. Kano, T. Tada and Y. Majima, Chem. Soc. Rev., 2015, 44, 970-987.

4 F. Sangghaleh, I. Sychugov, Z. Y. Yang, J. G. C. Veinot and J. Linnros, ACS Nano, 2015, 9, 7097-7104.

5 X. Y. Cheng, S. B. Lowe, P. J. Reece and J. J. Gooding, Chem. Soc. Rev., 2014, 43, 2680-2700.

6 V. Singh, Y. X. Yu, Q. C. Sun, B. Korgel and P. Nagpal, Nanoscale, 2014, 6, 14643-14647.

7 C. Leendertz, V. S. Chirvony, R. García-Calzada, L. Görög, J. A. Töfflinger, L. Korte, S. Agouram, J. Martínez-Pastor, N. Petermann, H. Wiggers and A. G. Ulyashin, Phys. Status Solidi A, 2015, 212, 156-161.

8 B. F. P. McVey and R. D. Tilley, Acc. Chem. Res., 2014, 47, 3045-3051.

9 Z. Bisadi, M. Mancinelli, S. Manna, S. Tondini, M. Bernard, A. Samusenko, M. Ghulinyan, G. Fontana, P. Bettotti, F. Ramiro-Manzano, G. Pucker and L. Pavesi, Phys. Status Solidi, 2015, 212, 2659-2671.

10 Y. Li, P. C. Humphreys, G. J. Mendoza and S. C. Benjamin, Phys. Rev. X, 2015, 5, 041007.

11 K. K. Likharev, Proc. IEEE, 1999, 87, 606-632.

12 T. Baron, P. Gentile, N. Magnea and P. Mur, Appl. Phys. Lett., 2001, 79, 1175-1177.

13 C. H. Cho, B. H. Kim and S. J. Park, Appl. Phys. Lett., 2006, 89, 013116.

14 B. Zaknoon, G. Bahir, C. Saguy, R. Edrei, A. Hoffman, R. A. Rao, R. Muralidhar and K. M. Chang, Nano Lett., 2008, 8, 1689-1694.

15 O. Wolf, M. Dasog, Z. Yang, I. Balberg, J. G. C. Veinot and O. Millo, Nano Lett., 2013, 13, 2516-2521.

16 C. Schönenberger, H. van Houten, J. M. Kerkhof and H. C. Donkersloot, Appl. Surf. Sci., 1993, 67, 222-227.

17 D. Anselmetti, T. Richmond, A. Baratoff, G. Borer, M. Dreier, M. Bernasconi and H.-J. Güntherodt, Europhys. Lett., 1994, 25, 297-302.

18 H. Zhang, Y. Yasutake, Y. Shichibu, T. Teranishi and Y. Majima, Phys. Rev. B: Condens. Matter Mater. Phys., 2005, 72, 205441.

19 H. Koo, S. Kano, D. Tanaka, M. Sakamoto, T. Teranishi, G. J. Cho and Y. Majima, Appl. Phys. Lett., 2012, 101, 083115 .
20 P. Liljeroth, L. Jdira, K. Overgaag, B. Grandidier, S. Speller and D. Vanmaekelbergh, Phys. Chem. Chem. Phys., 2006, 8, 3845-3850.

21 M. V. Wolkin, J. Jorne, P. M. Fauchet, G. Allan and C. Delerue, Phys. Rev. Lett., 1999, 82, 197-200.

22 J. P. Wilcoxon, G. A. Samara and P. N. Provencio, Phys. Rev. B: Condens. Matter Mater. Phys., 1999, 60, 2704-2714.

23 S. M. Prokes, W. E. Carlos and O. J. Glembocki, Phys. Rev. B: Condens. Matter Mater. Phys., 1994, 50, 17093-17096.

24 V. I. Klimov, J. Phys. Chem. B, 2000, 104, 6112-6123.

25 P. Becker, K. Dorenwendt, G. Ebeling, R. Lauer, W. Lucas, R. Probst, H. J. Rademacher, G. Reim, P. Seyfried and H. Siegert, Phys. Rev. Lett., 1981, 46, 1540-1543.

26 P. Podsiadlo, G. Krylova, B. Lee, K. Critchley, D. J. Gosztola, D. V. Talapin, P. D. Ashby and E. V. Shevchenko, J. Am. Chem. Soc., 2010, 132, 8953-8960.

27 P. Podsiadlo, B. Lee, V. B. Prakapenka, G. V. Krylova, R. D. Schaller, A. Demortière and E. V. Shevchenko, Nano Lett., 2011, 11, 579-588.

28 C. Xiao, J. Guo, P. Zhang, C. Chen, L. Chen and L. M. Qian, Sci. Rep., 2017, 7, 40750.

29 I. Horcas, R. Fernández, J. M. Gómez-Rodriguez, J. Colchero, J. Gómez-Herrero and A. M. Baro, Rev. Sci. Instrum., 2007, 78, 013705.

30 A. E. Hanna and M. Tinkham, Phys. Rev. B: Condens. Matter Mater. Phys., 1991, 44, 5919-5922.

31 U. Banin and O. Millo, Annu. Rev. Phys. Chem., 2003, 54, 465-492.

32 Y. M. Niquet, C. Delerue, G. Allan and M. Lannoo, Phys. Rev. B: Condens. Matter Mater. Phys., 2002, 65, 165334.

33 M. Lannoo, C. Delerue and G. Allan, Phys. Rev. Lett., 1995, 74, 3415-3418.

34 C. Y. Ng, T. P. Chen, L. Ding, Y. Liu, M. S. Tse, S. Fung and Z. L. Dong, Appl. Phys. Lett., 2006, 88, 063103.

35 L. W. Wang and A. Zunger, Phys. Rev. Lett., 1994, 73, 10391042.

36 A. Franceschetti and A. Zunger, Phys. Rev. B: Condens. Matter Mater. Phys., 2000, 62, 2614-2623.

37 K. Kůsova, P. Hapala, J. Valenta, P. Jelínek, O. Cibulka, L. Ondič and I. Pelant, Adv. Mater. Interfaces, 2014, 1, 1300042.

38 D. M. Fleetwood, P. S. Winokur, R. A. Reber, T. L. Meisenheimer, J. R. Schwank, M. R. Shaneyfelt and L. C. Riewe, J. Appl. Phys., 1993, 73, 5058-5074.

39 N. Armbrust, F. Schiller, J. Güdde and U. Höfer, Sci. Rep., 2017, 7, 46561.

40 E. Burzurí and H. S. J. van der Zant, in Molecular Magnets: Physics and Applicatios, ed. J. Bartolome, F. Luis and J. F. Fernandez, Springer-Verlag, Germany, 1st edn, 2014, ch. 12, pp. 297-317.

$41 \mathrm{~J}$. C. Cuevas and E. Scheer, Molecular Electronics: An Introduction to Theory and Experiment, World Scientific, Singapore, 2010, vol. 1.

42 S. Godefroo, M. Hayne, M. Jivanescu, A. Stesmans, M. Zacharias, O. I. Lebedev, G. Van Tendeloo and V. V. Moshchalkov, Nat. Nanotechnol., 2008, 3, 174-178. 
43 H. Heckler, D. Kovalev, G. Polisski, N. N. Zinov'ev and F. Koch, Phys. Rev. B: Condens. Matter Mater. Phys., 1999, 60, 7718-7721.

44 Z. T. Xie, S. Shi, F. L. Liu, D. L. Smith, P. P. Ruden and C. D. Frisbie, ACS Nano, 2016, 10, 8571-8577.

45 S. Sasaki, S. De Franceschi, J. M. Elzerman, W. G. van der Wiel, M. Eto, S. Tarucha and L. P. Kouwenhoven, Nature, 2000, 405, 764-767.

46 J. G. A. Dubois, E. N. G. Verheijen, J. W. Gerritsen and H. van Kempen, Phys. Rev. B: Condens. Matter Mater. Phys., 1993, 48, 11260-11264.
47 J. G. Simmons, J. Appl. Phys., 1963, 34, 1793-1803.

48 J. Wang, S. Q. Sun, F. Peng, L. X. Cao and L. F. Sun, Chem. Commun., 2011, 47, 4941-4943.

49 X. Y. Cheng, R. Gondosiswanto, S. Ciampi, P. J. Reece and J. J. Gooding, Chem. Commun., 2012, 48, 1187411876.

50 C. Debuschewitz, F. Münstermann, V. Kunej and E. Scheer, J. Low Temp. Phys., 2007, 147, 525-535.

51 M. Wolz, C. Debuschewitz, W. Belzig and E. Scheer, Phys. Rev. B: Condens. Matter Mater. Phys., 2011, 84, 104516. 